\title{
Modern Mild Skin Cleansing
}

\section{Zhengyuan Li}

Mill Hill School, Burton Bank Wills Grove, Mill Hill Village, London, UK

Email: laurindali@163.com

How to cite this paper: Li, Z.Y. (2020) Modern Mild Skin Cleansing. Journal of Cosmetics, Dermatological Sciences and Applications, 10, 85-98. https://doi.org/10.4236/jcdsa.2020.102009

Received: February 6, 2020

Accepted: June 12, 2020

Published: June 15, 2020

Copyright (c) 2020 by author(s) and Scientific Research Publishing Inc. This work is licensed under the Creative Commons Attribution International License (CC BY 4.0).

http://creativecommons.org/licenses/by/4.0/

\begin{abstract}
Surfactant, an abbreviation for the surface-active agent, is utilized in almost every industry. It brings two immiscible phases such as oil and water into one single homogeneous phase, leading to various industrial applications such as food, painting, coating, drug delivery as well as cosmetics. The use of surfactants in skin cleansing is very common to keep skin healthy. Their function herein is to lower the interfacial tension at the dirt/water and skin/water interfaces, thereby detaching dirt, extra sebum or oils from the skin surface. But this application could bring side effects attributed to the penetration of surfactants into the skin, including skin proteins denaturation, stratum corneum lipids removal or even lipids organization disruption in the stratum corneum. This review summarizes modern mild skin cleansing technologies, which address the side effects brought by the surfactants.
\end{abstract}

\section{Keywords}

Mild Cleansing, Skin, Surfactant, Micelle, Polymer

\section{Introduction}

Nowadays, people pay more attention to their beauty. A woman usually wears makeup every day, therefore the proper cleansing process afterward is important, making skin healthy and comfortable.

To keep skin healthy, it is essential to keep the hygiene of the skin by daily removing dirt, extra sebum, and oils from the skin surface. Since several decades ago, soap has been one of the most popular cleansing products thanks to its high foaming ability and strong cleansing power, but there also have been reports of soap-induced skin irritations such as dry and tight skin [1]-[8]. To avoid aggressive skin cleansing, people currently tend to prefer mild cleansers, which can minimize the skin barrier damage. Nevertheless, to the best of our knowledge, there has been a lack of literature summary of modern mild skin cleansing technologies. To address this issue, a general overview will be given first about the skin 
structure such as epidermis structure and surfactants including their classifications; followed by a summary of the proposed surfactant penetration models which are the surfactant monomer penetration model, the surfactant micelle penetration model and the charge density correlation model; a simple overview of the two-stage surfactant-protein interaction and the three possible mechanisms of how surfactants interact with skin lipids; and finally the current approaches to the formulation of mild cleansers based on the proposed surfactant skin penetration models and possible mechanisms of surfactant interactions with skin proteins and lipids discussed above [6] [9] [10] [11] [12].

\section{Skin Structure}

The skin structure can be divided into three parts: hypodermis, dermis and epidermis as shown in Figure 1. The hypodermis is the third layer of the skin, and is an elastic layer and includes a large amount of fat cells that work as a shock absorber for blood vessels [13]. The dermis which is above the hypodermis, comprises blood vessels, hair follicles, sebaceous glands, and sweat glands [14]. The epidermis is the top layer of the skin, and it is made up of both non-viable epidermis and viable epidermis (Figure 2). The non-viable epidermis is the stratum corneum (SC), which can be considered as the barrier to penetration. The layers below the stratum corneum are viable epidermis which consist of blood capillaries and nerve fibers. 95\% of the total cells in the epidermis are keratinocytes [15].

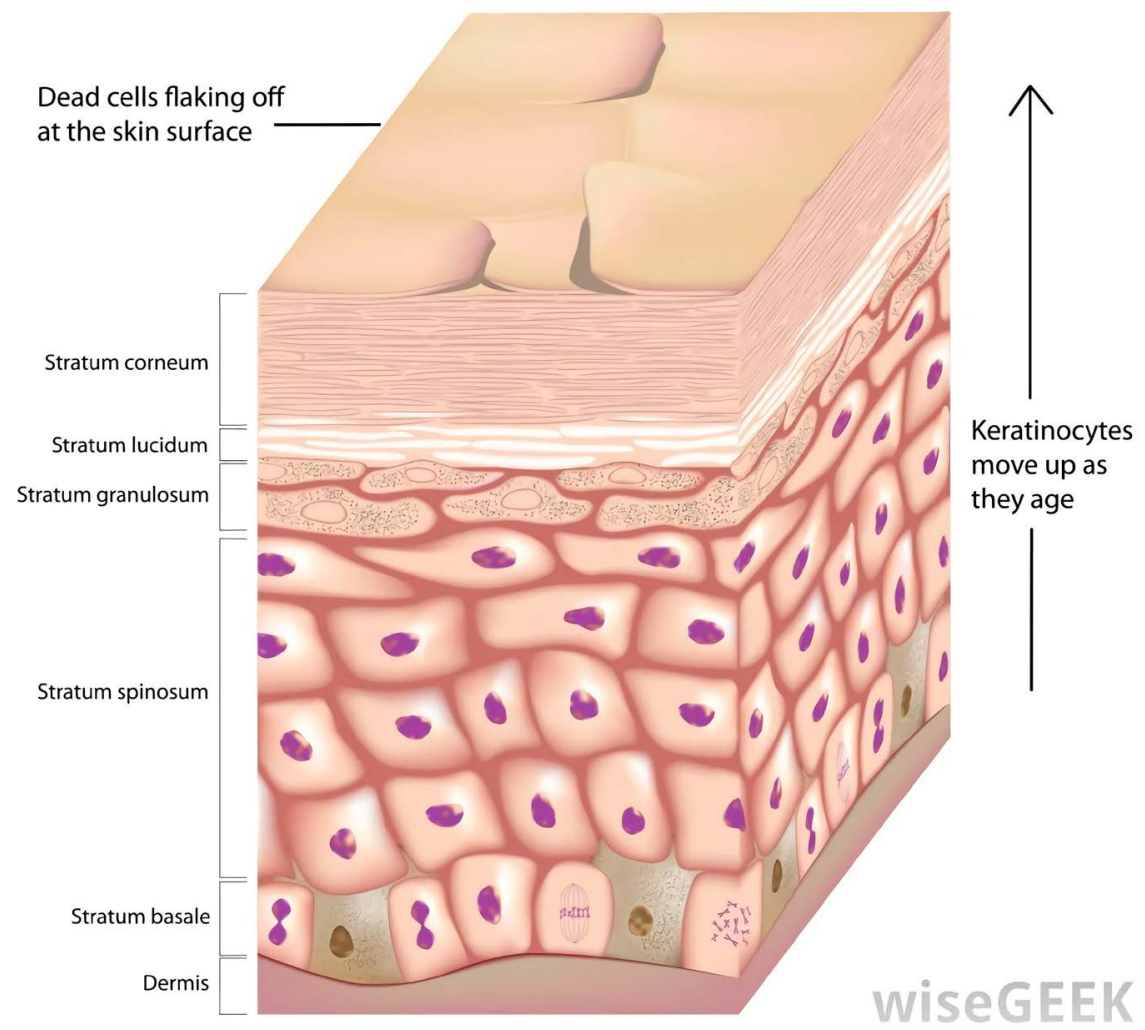

Figure 1. The skin is composed of three main layers: the epidermis, dermis and the hypodermis [15]. 


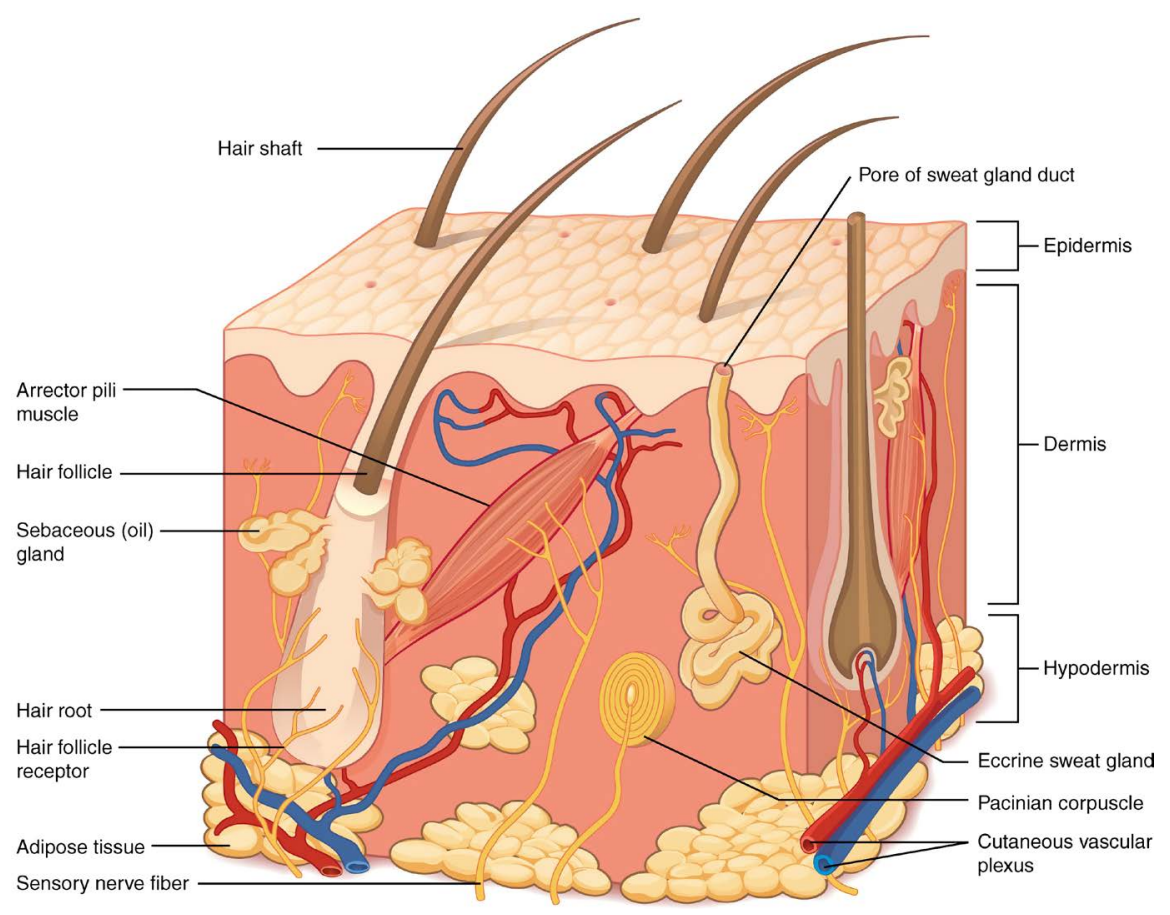

Figure 2. The structure of skin epidermis [20].

The structure of the stratum corneum can be described as "bricks and mortar". There are corneocytes and intercellular lipids in the SC, where the "bricks" are representing the corneocytes and "mortar" is the intercellular lipids [16] [17] [18] [19]. The SC is composed of approximately $40 \%$ protein, mostly keratin [20]. Cleanser surfactants can bind to SC proteins, leading to transient swelling and hyperhydration under washing conditions. As water evaporates after washing, SC proteins will be shrinking, leading to a drying stress [21].

The skin lipids have occupied approximately $10 \%$ to $15 \%$ of the SC. The major lipids in the SC are ceramides, which approximately occupied $47 \%$. There are also cholesterols, fatty acids and cholesterol esters [20]. The physical conformation of the intercellular lamellar lipids provides a tight and semipermeable barrier to the passage of water through the tissue. The structural organization as illustrated in Figure 3 is proposed to account for these observations. The simultaneous presence of both a crystalline and a liquid crystalline phase is depicted by the domain mosaic model of SC lipid organization. The fluid phase allows flexibility within the lipid layer without compromising permeability barriers of the SC [22]. The packing of the lipids within the bilayers is expected to control the overall barrier properties. Orthorhombic, hexagonal and fluid bilayers are all proposed forms of bilayers, among which orthorhombic is the most compact packing with the highest barrier properties while the fluid form is the least [22] [23].

\section{An Introduction to Surfactant}

Surfactant, an abbreviation for surface active agent, is an amphiphilic molecule that has both hydrophilic and hydrophobic/lipophilic parts. In cosmetics, surfactant 




Figure 3. The skin lipids structural organization [24].

is commonly used as a detergent in facial cleansing. The function herein is to lower the interfacial tension at the dirt/water and skin/water interfaces, thereby detaching the dirt from the skin surface. The dirt can also be removed in an emulsified form [24]. Surfactants tend to form aggregates called micelles, where the surfactants hydrophobic groups are directed towards the interior of the cluster and the polar heads are directed towards water. The micelle is a polar aggregate of high-water solubility without much surface activity. It is only the surfactant monomers that are capable of lowering surface tension [1] [6] [7] [24] [25] [26] [27].

Surfactants can be classified into four groups based on the charge presented on the polar groups after dissociation in the aqueous solution: anionic, cationic, non-ionic and amphoteric.

\section{Anionic Surfactant}

Anionics are the most common used surfactant, occupying about $60 \%$ annual worldwide surfactant production. Their hydrophilic heads possess negative charges. Anionic surfactants have strong cleansing power, good wetting properties and excellent lather characteristic, but they are considered to be irritated to the skin and eyes [1] [2]. The examples of the most commonly used anionic surfactants are soap, sodium lauryl sulphate (SLS), and sodium laureth sulphate (SLES). Soap is still the largest single type of surfactant, which is a generic name representing the alkali metal salts of carboxylic acids derived from animal fats or vegetable oils. 
Perhaps the most famous soap cleanser on the market is Shiseido Senka Perfect Whip Cleansing Foam. SLS is a well-known wetting agent and a detergent, which has been proven to irritate the skin leading to the skin barrier damage as well. SLES is the primary surfactant in commercial body wash, shower gel and shampoo, owing to its good cleansing power and low cost. The commercial examples containing SLES are Lush I Love Juicy Shampoo and La Roche-Posay Effaclar Purifying Foaming Gel Cleanser. However, SLES also has some irritant potential to the skin [3] [4] [5]. Anionic surfactant is normally used as a primary surfactant, thanks to its moderate cost, and more importantly the irritation potential can be reduced in the formulation by mixing the anionic surfactant with polymers and other types of surfactants [6] [7] [8].

\section{Cationic Surfactant}

Cationic surfactants are positively charged, and have lower deterging power than that of anionic surfactants, however, they can be used as antimicrobial preservatives due to the considerable bactericidal activity against a wide range of microorganisms [26]. The examples of the most commonly used cationic surfactant are amine salts and quaternary ammonium salts [28]. For example, Cetrimonium Bromide is used in Bioderma Sensibio Micellar Water.

\section{Nonionic Surfactant}

Nonionic surfactant is the second largest surfactant groups, and has no electrical charge on its head, so it is normally compatible with all other types of surfactant. The nonionic surfactants are also used as thickeners for shampoos, as emulsifiers and suspending agents in cosmetics, pharmaceuticals and foods [29]. The nonionic surfactants are regarded as the lowest irritants to the skin [2], which is why they are often formulated in eye makeup removers such as PEG-6 Caprylic/capric glycerides in Bioderma Sensibio Micellar Water. The physicochemical properties of the ethoxylated compounds are very temperature dependent. Non-ionic surfactants containing polyoxyethylene chain exhibit reverse solubility versus temperature in water. As temperature rises, the interactions between the oxyethylene groups and water become weaker, therefore two phases eventually appear. The temperature at which it occurs is called the cloud point. The cloud point mainly depends on the number of oxyethylene units while the hydrophobic chain length has a less influence [2].

\section{Amphoteric surfactant}

Amphoteric surfactants usually contain two charged groups with different signs. An amphoteric surfactant is one that changes from net cationic via zwitterionic to net anionic ongoing from low $\mathrm{pH}$ to high $\mathrm{pH}$. The change in $\mathrm{pH}$ naturally affects surfactant properties such as foaming, wetting, detergency and etc. Amphoteric surfactants are mild to skin and also exhibit low eye irritation and are frequently used in shampoos and other cosmetic products. For instance, Coco-betaine was formulated together with SLES in La Roche-Posay Effaclar Purifying Foaming Gel Cleanser to improve its skin mildness. However, their application sometimes is restricted by their high costs [30]. 


\section{General physicochemical properties of surfactant}

There are many physicochemical properties of surfactants, and one of the most important characteristics is the critical micelle concentration (CMC). CMC is the concentration of the surfactant above which micelles are spontaneously formed [31]. When the concentration is below the CMC, the surfactant molecule that has been added into the water will arrange on the air-water interface, with the hydrophobic part facing toward the air. As the added surfactant concentration reaches a certain level, there is no space for the rest surfactant to be placed at the interface, therefore, the molecules start to form aggregates, which is called micelles. Any further addition of the surfactants after reaching CMC will only increase the number of micelles in the aqueous phase. The surfactant molecule that has been placed on the interface is called monomer, and only monomers have surface activity, meaning only monomers could lower the surface tension between two phases, so beyond the CMC, there is no further reduction in the interfacial tension. Usually anionic and cationic surfactants have higher CMCs than those of nonionic surfactants, due to the fact that the charge degree of the hydrophilic group could influence the CMC value [10] [28] [30].

Another interesting characteristic of the surfactant is the packing geometry. The surfactant structure formed is as a result of the balance between the polar and the non-polar part of the surfactant molecule. The critical packing parameter (CPP) can be seen as the ratio between the cross-sectional area of the hydrophobic part and that of the hydrophilic head part. When the CPP is smaller than 1 , it can be arranged as a normal micelle, normal discontinuous cubic, normal hexagonal, or normal bicontinuous cubic. When the CPP is equal to 1 , it usually forms a lamellar phase. When the CPP is greater than 1, it can form reverse hexagonal, reverse discontinuous cubic, or reverse micelle [32].

\section{Surfactant Skin Penetration Models}

There are three generally accepted models that have partially explained the mechanisms behind surfactant penetrating into skin.

\section{The Surfactant Monomer Skin Penetration Model}

The monomer penetration model is the most commonly accepted model, as it regards that only surfactant monomers could penetrate into the skin, either because that only surfactant monomers are surface active or the micelles are too big to pass through the skin barrier [1] [6] [33]. This view is based on experimental observations which a correlation between the amount of surfactant monomers that have been added in and the damage level of the skin has been shown [12] [33] [34]. It is widely accepted that the surfactants need to penetrate into the SC and then induce irritation. However, if the surfactant monomer skin penetration model is always true, the level of penetrated surfactant should reach a maximum, which is at the CMC of the surfactant. As a result, surfactant concentration beyond the CMC would have no effects on the contribution of surfactant-induced skin irritation. However, it is well-known that the surfactant-induced skin irritation exacerbates as the total surfactant concentration increases beyond the CMC [12]. 


\section{The Surfactant Micelle Skin Penetration Model}

To rationalize the observed dose-dependent effect of the surfactant-induced irritation, researchers proposed that: 1) the monomer activity increased beyond the CMC, and 2) the micelles solubilized lipids present in the skin. In contrast, it was found out that the surfactant monomer activity does not increase but slightly decreases beyond the CMC. In addition, an increase in the number of micelles above the CMC demonstrates an increase in the lipid solubilization capacity, but it could not explain the observed enhanced surfactant penetration into skin [31].

Therefore, a more complex mechanism has been proposed. It was suggested that not only surfactant monomers but also some micelles are able to penetrate into the skin [35]. Moore et al. [31] carried out an investigation whether an increase in the total SDS concentration above the CMC in the contacting solution would lead to an increase in the SDS concentration in the epidermis. The results showed that there was a linear relationship between the SDS concentration in the contacting solution and the SDS concentration in the epidermis, contradicting the view of surfactant monomer skin penetration model [31].

Surfactant micelle penetration model is thus proposed. To consider the micelle penetration model, it is necessary to consider the micelle size relative to the radius of aqueous pore in the skin barrier, therefore it is interesting to examine the effect of adding poly (ethylene oxide) (PEO) to the surfactant solution. In the presence of PEO, the SDS concentration in the epidermis has been reduced significantly [31]. This was due to that the PEO could bind to the SDS micelles, and form micelle-like complexes with SDS, which have a relatively larger size than that of the SDS micelles. The larger PEO-bonded micelles were postulated to be hindered by the aqueous pores, thereby reducing SDS skin penetration [31]. The researchers also studied SDS micelles skin penetration by mixing SDS with the ethylene oxide type non-ionic surfactants $\left(\mathrm{C}_{\mathrm{m}} \mathrm{E}_{\mathrm{n}}\right)$ [31], and they discovered that the SDS from the SDS/ $\mathrm{C}_{\mathrm{m}} \mathrm{E}_{\mathrm{n}}$ mixtures is less able to penetrate into the skin than that from the pure SDS solution. By using the dynamic light scattering technique, they revealed that the SDS/ $C_{m} E_{n}$ mixed micelles had larger sizes than those of the pure SDS micelles, and hence the increase in the micelle size reduced the surfactant penetration into the skin was proposed [31].

Glycerin is a well-known humectant and skin beneficial agent. An investigation on whether the addition of glycerin would reduce the SDS micelle skin penetration was carried out. It was shown that the addition of $10 \mathrm{wt} \%$ glycerin to the SDS solution did not reduce its CMC or micelle size. On the other hand, with the addition of $10 \mathrm{wt} \%$ glycerin, the size and the number density of the aqueous pores in the SC were reduced, suggesting that the SDS micelles were sterically hindered to penetrate [34].

Sodium cocoyl isethionate (SCI) is a very important surfactant that has been using in facial cleansers and body washes, attributed to its mild, less irritate characteristics to the skin. One of the reasons accounting for the mildness of SCI was the incapability of its micelles to contribute to the skin penetration. It was shown that the SCI micelles have a significant larger size than that of the skin 
aqueous pores, suggesting that it is unfavorable for the SCI micelles to penetrate into the skin and induce further irritation (Table 1) [36]. Ananthapadmanabhan et al. [29] observed that the CMC of SCI is lower than that of SDS, and binds to proteins only about one fifth as much as the SDS does under similar conditions and exposure time, which has proved that monomer penetration model is correct in this way [37].

\section{Charge Density Correlation Model}

The mechanisms of anionic surfactant penetration into skin are still widely debated. Neither the monomer penetration model nor the micelle penetration model could fully explain how anionic surfactant penetrates into the skin. A new hypothesis is proposed in which short-term penetration is based on monomer concentration and longer-term penetration is based on surfactant-induced damage to the skin barriers [36]. A strong correlation was found between the amount of penetrated anionic surfactants and the Zeta potential of the surfactant solutions. When an anionic surfactant solution contacts with the skin, monomers penetrate into the skin, bind to proteins and increase the charge on protein networking, leading to swelling of the structure. This allows progressive surfactant binding in even deeper skin layers, leading to further structural swelling and surfactant penetration [36]. As the damage to the skin increases, it is possible for micelles and small surfactant aggregates to penetrate, leading to further swelling of the structures. The more negative charged surfactant system, the longer the surfactant exposure time, more bindings could occur, resulting in more swelling of the structures. These structures are likely to have a charge characteristic proportional to micelle-like structures, which could explain the correlation between the Zeta potential and the micelle penetration. Interestingly, their data did not show a strong correlation between the micelle size and surfactant penetration. The less skin penetration of SDS micelles with PEO was hypothesized to be attributed to the lower diffusion speed [38].

\section{Interaction between Surfactant and Proteins/Lipids}

Protein are copolymers built up of amino acids that contain polar and non-polar groups. The polar groups cloud be ionic or non-ionic, which may also vary with $\mathrm{pH}$, thus protein can be seen as an amphoteric polyelectrolyte with some hydrophobic groups or as an amphiphilic polymer with a variable charge density. It has occupied approximately $40 \%$ of the SC [31].

Table 1. Skin aqueous pore characteristic resulting from skin exposure to three solutions [32].

\begin{tabular}{ccccc}
\hline $\begin{array}{c}\text { Types of aqueous } \\
\text { contacting } \\
\text { solution }\end{array}$ & $\begin{array}{c}\text { Average aqueous } \\
\text { pore radius } \\
\text { Rpore (A) }\end{array}$ & $\begin{array}{c}\text { Normalized pore } \\
\text { number density } \\
(\mathrm{E} / \mathrm{T}) \text { normal }\end{array}$ & $\begin{array}{c}\text { Micelle radius } \\
\text { (A) }\end{array}$ & $\begin{array}{c}\text { Critical micelle } \\
\text { concentration, } \\
\text { CMC (mM) }\end{array}$ \\
\hline a) SCI & $29 \pm 5$ & $2 \pm 1$ & $33.5 \pm 1$ & 1.0 \\
b) SDS & $33 \pm 5$ & $7 \pm 1$ & $19.5 \pm 1$ & 8.7 \\
c) PBS Control & $20 \pm 3$ & 1 & N/A & N/A
\end{tabular}


There are two stages of binding between surfactant and water-soluble protein: site-specific binding and co-operative binding. Site-specific binding is the surfactant binds to sites on the protein for which there is favourable interaction between the surfactant and the site. An example for this is the binding of an anionic surfactant to positively charged amino acid group on the protein. The sites on the protein relative to the total number of side chains, which forms one-to-one specific interactions [39].

Surfactants such as non-ionic surfactants do not go through site specific interactions with the protein, due to the fact that interactions are not free energetically favourable. Those surfactants interact with the protein through another stage which is called cooperative binding. In cooperative binding, the surfactant molecules aggregate to form micellar structures that interact with the protein. This interaction takes place and corresponds to the protein unfolding and losing its secondary structure [39].

To reduce the protein bindings, a bulky non-ionic surfactant is favorable due to: 1) the protein backbone shields exposed hydrophobic patches at the hydrophobic core-water micellar interface from the aqueous environment surrounding the micelle. A large head group will shield and reduce the hydrophobic surface area of the micelle core exposed to water, thus reducing the driving force for protein backbone to bind to the surface, by increasing the steric interactions between the protein and the surfactant heads; 2) the hydrophobic protein side chains are able to penetrate into the hydrophobic core of the micelle. The large hydrophilic heads of the non-ionic surfactants should also sterically hinder the access of the hydrophobic protein side chains into the micellar interior, thus reducing the strength of the interactions. In addition, it has been found that the reduction in the micelle surface charge density could make the electrostatic interactions less favourable, thereby mixing anionic surfactants with non-ionic surfactants is a common method to reduce surfactant bindings to proteins [39].

It is recognized that the lipid organization controls the skin absorption of exogenous substances, therefore, any damage to the lipid organization could lead to an alteration of the skin barrier [32]. The skin lipid phases consist in a lamellar structure made of stacks of lipid bilayers. Fluid phase is the most permeable phase in their molten state, whereas there are two solid crystalline phases: the orthorhombic phase (OR) and the hexagonal phase (HEX) [28]. As the damage to the skin barrier increases, the organization of the lipids will be more likely shifted to the liquid crystalline phase [40].

There are three possible mechanisms of surfactants interacting with the SC lipids: 1) removal of the surface lipids by the detergent action of surfactants; 2) disorganization of the SC by mixing with surfactants. This happens when the surfactants mix with lipids in the SC, which will distort the organization of the lipids; 3 ) solubilization and extraction of the SC lipids into the surfactant solution. The surfactant monomers penetrate into the SC and insert in the lipid bilayer followed by micellar solubilization of cholesterol, free fatty acids and fatty esters [41]. 
Investigation was carried on about how different types of surfactant contributed to the removal of different types of skin lipids. SLS, sodium stearoyl lactylate (SSL), cetyl trimethyl ammonium chloride (CTAC), distearyldimonium chloride (DC), laureth-23, PEG-25 hydrogenerated castor oil, PEG-100 stearate, PEG-12 dimenthicone, Polyoxyethylene sorbitan laurate (polysorbate) and hydrogenated lecithin were examined to remove various model skin lipids such as squalene, triglycerides (TG), ceramides and cholesterol [41]. The results showed that different surfactants can remove different types of lipids in the skin. For SLS, there was a significant decrease in squalene and n-alkane; for SLS and CTAC, there was a significant decrease of TG. All surfactants induced an increase of the polar component of the skin except PEG-12 dimethicone and polysorbate. SLS, CTAC, and DC were significant on removing ceramides and TG. Ceramide levels became less than 3 times lower than TG after the surfactant treatment. No surfactant removed a significant high level of cholesterol [41]. PEG-12 dimethicone and polysorbate are exception of inducing an increase of the polar components in the skin, indicating they are mild to the skin. The structural transition of lipid bilayer from the orthorhombic phase to the hexagonal phase was detected whereas the disorganization to the liquid crystalline phase was not observed. Polysorbate and PEG-12 dimethicone were identified as the mildest surfactants in this study, probably thanks to their large PEG headgroups, suggesting that bulky PEG headgroup inhibited the penetration of these surfactants into the SC lipid matrix [40].

\section{Modern Mild Cleanser Formulation}

Recent researches and applications have provided more information on the interactions between surfactants and skin, which helps the industry develop technologies that can produce cleansers that are both functional and mild. Liquid cleansers usually have a combination of anionic and amphoteric surfactants along with high levels of oils and occlusive as moisturizers, in order to reduce the protein denaturation and skin barrier damage [29]. However, the addition of amphoteric surfactants cannot solve the problem completely, a certain degree of skin lipids damage still occurs. The ratio between anionic and amphoteric surfactants also needs to be considered. The current moisturizing shower gels usually use moisturizing oils and humectants at a very high concentration, even though most of them will be washed away during rinsing [37]. Nowadays, the cleansers often use a combination of mild surfactant systems and efficient natural emollient oils, lipids, occlusive, as well as humectants.

Skin dryness is one of the common problems caused by cleansers, and it is usually addressed by occlusives, emollients and/or humectants. Petrolatum act as an occlusive barrier, reducing the evaporation of the water inside the skin. Liquid triglyceride oils can penetrate into skin cracks and crevices, and moisturize the deeper skin layers. More importantly it can also prevent surfactants from interacting with the skin proteins [37]. Glycerol is used as a humectant to retain 
skin moisture, however, due to its high-water solubility, the addition of glycerol in cleansers becomes inefficient, because a large amount of glycerol will be lost during wash. Simple addition of petrolatum and triglycerides in cleansers is facing this problem as well. The industry uses long-chain fatty acids (C16-C18) to lower the tendency of a surfactant system to damage lipid membranes. This is perhaps due to their ability to act as a buffer against lipid extraction by surfactant micelles, and to deposit and to replenish some of the fatty acid lipids lost during cleansing, which makes the cleanser milder [21].

The use of polymers to make milder cleansers is an alternative approach. Polyethylene oxide (PEO) has been shown to alter micelles and reduce the aggressiveness of surfactants [42]. The PEO chains bind water molecules and the surfactant monomers, increasing the sizes of the micelles, thereby sterically hindering the micelle penetration into skin. More recently, hydrophobically modified polymers (HMPs) have been used instead of PEO. HMPs can associate with surfactants in solutions. Surfactant self-assembled to the hydrophobic domains of the HMPs, results in slower surfactant dynamics [37]. In addition, thanks to the larger sizes of the surfactants, less surfactants can penetrate into the SC, making the cleanser less aggressive. A gentle facial cleanser with HMPs was developed by researchers in Johnson \& Johnson, and the clinical test results demonstrated that HMPs do minimize the SC disruption [37].

\section{Conclusion and Perspectives}

The focus of this review paper is to summarize the state of the art understandings of modern mild skin cleansing technologies. Anionic surfactants have good lathering and cleansing properties making them the ideal ingredients in cleanser formulations, on the other hand, anionic surfactants have small micelle size and high charge density, potentially contributing to the skin irritation induced by surfactants. To address this issue, addition of non-ionic/amphoteric surfactants to the cleanser formulation can reduce the micelle charge density and sterically hinder the micelle-skin protein binding, making the cleanser less harsh to the skin. In addition, adding polymers to the surfactant system further increases the micelle size and decreases the micelle diffusion speed, thereby hindering surfactant penetrating into skin. Last but not the least, incorporation of humectants, emollients as well as occlusive into the surfactant system in the cleanser formulation can either reduce the surfactant skin penetration, or lower the surfactant system damaging the skin lipids, thereby minimizing the occurrence of skin irritation.

\section{Acknowledgements}

The author is grateful for the opportunities provided by Mill Hill School in London, UK and the ViaX Online Education Platform.

\section{Conflicts of Interest}

The author declares no conflicts of interest regarding the publication of this paper. 


\section{References}

[1] Ananthapadmanabhan, K.P., Yu, K.K., Meyers, C.L. and Aronson, M.P. (1996) Binding of Surfactants to Stratum Corneum. Journal of the Society of Cosmetic Chemists, 47, 185-200.

[2] Strube, D.D., Koontz, S.W., Murahata, R.I. and Theiler, R.F. (1989) The Flex Wash Test: A Test Method for Evaluating the Mildness of Personal Washing Products. Journal of the Society of Cosmetic Chemists, 40, 297-306.

[3] Imokawa, G. (1997) Surfactant Mildness. In: Rieger, M.M. and Rhein, L.D., Eds., Surfactants in Cosmetics, Marcel Dekker, New York, 427-471.

[4] Scheuplein, R. and Blank, I. (1971) Permeability of the Skin. Physiological Reviews, 51, 702-747. https://doi.org/10.1152/physrev.1971.51.4.702

[5] Abraham, W. (1997) Surfactant Effects on Skin Barrier. In: Rieger, M.M. and Rhein, L.D., Eds., Surfactants in Cosmetics, Marcel Dekker, New York, 437-487.

[6] Faucher, J.A. and Goddard, E.D. (1978) Interaction of Keratinous Substrates with Sodium Lauryl Sulfate. I. Sorption. Journal of the Society of Cosmetic Chemists, 29, 323-337.

[7] Faucher, J.A. and Goddard, E.D. (1978) Interaction of Keratinous Substrates with Sodium Lauryl Sulfate. II. Permeation through Stratum Corneum. Journal of the Society of Cosmetic Chemists, 29, 339-353.

[8] Rhein, L.D. and Sireion, F.A. (1991) Surfactant Interactions with Skin. In: Bender, M., Ed., Interfacial Phenomena in Biological Systems, Surfactant Science Series, Vol. 39, CRC Press, Boca Raton, 33-49.

[9] Hama, I., Sasamoto, H., Tamura, T., Nakamura, T. and Miura, K. (1998) Skin Compatibility and Ecotoxicity of Ethoxylated Fatty Methyl Ester Nonionics. Journal of Surfactants and Detergents, 1, 93-97. https://doi.org/10.1007/s11743-998-0012-8

[10] Rhein, L.D., Robbins, C.R., Fernee, K. and Cantore, R. (1986) Surfactant Structure Effects on Swelling of Isolated Human Stratum Corneum. Journal of the Society of Cosmetic Chemists, 37, 125-139.

[11] Imokawa, G., Sumura, K. and Katsumi, M. (1975) Study on Skin Roughness Caused by Surfactants: II. Correlation between Protein Denaturation and Skin Roughness. Journal of the American Oil Chemists' Society, 52, 484-489. https://doi.org/10.1007/BF02640737

[12] Hall-Manning, T.J., Holland, G.H., Rennie, G., Revell, P., Hines, J., Barratt, M.D. and Basketter, D.A. (1998) Skin Irritation Potential of Mixed Surfactant Systems. Food and Chemical Toxicology, 36, 233-238. https://doi.org/10.1016/S0278-6915(97)00144-0

[13] Evert, R.F. (2006) Esau's Plant Anatomy: Meristems, Cells, and Tissues of the Plant Body-Their Structure, Function, and Development. 3rd Edition, Wiley, Hoboken, NJ. https://doi.org/10.1002/0470047380

[14] Keleb, E., Sharma, R.K., Mosa, E.B. and Aljahvi, A.Z. (2010) Transdermal Drug Delivery System-Design and Evaluation. International Journal of Advances in Pharmaceutical Sciences, 1, 201-211.

[15] Lumenlearning, Module 24: The Integumentary System, Structure and Function of the Skin.

https://courses.lumenlearning.com/wmopen-biology2/chapter/structure-and-functi on-of-skin/

[16] Elias, P.M. (1981) Lipids and the Epidermal Permeability Barrier. Archives of Dermatological Research, 270, 95-117. https://doi.org/10.1007/BF00417155

[17] Wertz, P.W. and Downing, D.E. (1989) Stratum Corneum: Biological and Bio- 
chemical Considerations. In: Hadgraft, J. and Guy, R.H., Eds., Transdermal Drug Delivery: Developmental Issues and Research Initiatives, Marcel Dekker, New York, $1-22$.

[18] Bronaugh, R.L. and Maibach, H.I. (1985) In Vitro Models for Human Percutaneous Absorption. In: Maibach, H.I. and Lowe, N.J., Eds., Models in Dermatology, Vol. 2, Karger, Basel, 178-188.

[19] Heisig, M., Lieckfeldt, R., Witturn, G., Mazurkevich, G. and Lee, G. (1996) Non Steady-State Descriptions of Drug Permeation through Stratum Corneum. I. The Biphasic Brick-and-Mortar Model. Pharmaceutical Research, 13, 421-426. https://doi.org/10.1023/A:1016048710880

[20] https://images.wisegeek.com/anatomy-of-the-epidermis.jpg

[21] Lips, A., Ananthapadmanabhan, K.P., Vethamuthu, M., et al. (2006) Role of Surfactant Micelle Charge in Protein Denaturation and Surfactant Induced Skin Irritation. In: Rhein, L.D., Schlossman, M., O’Lenick, A. and Somasundaran, P., Eds., Surfactants in Personal Care Products and Decorative Cosmetics, CRC Press, Boca Raton, 177-187.

[22] Ananthapadmanabhan, K.P., et al. (2009) A Novel Technology in Mild and Moisturizing Cleansing Liquids. Cosmetic Dermatology, 22, 307-316.

[23] Vreeken, R.J. https://www.researchgate.net/figure/Lamellar-and-lateral-organization-in-human stratum-corneum-1-The-outermost-layer-of_fig4_231613918

[24] Vilaplana, J., Mascaro, J.M., Trullas, C., Coil, J., Romaguera, C., Zemba, C. and Pelejero, C. (1992) Human Irritant Response to Different Qualities and Concentrations of Cocoamidopropylbetaines: A Possible Model of Paradoxical Irritant Response. Contact Dermatitis, 26, 289-294.

https://doi.org/10.1111/j.1600-0536.1992.tb00120.x

[25] Brito, R.O., Silva, S.G., Fernandes, R.M., Marques, E.F., Enrique-Borges, J. and do Vale, M.L. (2011) Enhanced Interfacial Properties of Novel Amino Acid-Derived Surfactants: Effects of Headgroup Chemistry and of Alkyl Chain Length and Unsaturation. Colloids and Surfaces B: Biointerfaces, 86, 65-70. https://doi.org/10.1016/j.colsurfb.2011.03.017

[26] Wilhelm, K.-P., Cua, A.B., Wolff, H.H. and Maibach, H.I. (1993) Surfactant-Induced Stratum Corneum Hydration in Vivo: Prediction of the Irritation Potential in Anionic Surfactants. Journal of Investigative Dermatology, 101, 310-315.

[27] Loden, M. (1990) The Simultaneous Penetration of Water and Sodium Lauryl Sulfate through Isolated Human Skin. Journal of the Society of Cosmetic Chemists, 41, 227-233.

[28] Robbins, C.R. and Fernee, K.M. (1983) Some Observations on the Swelling Behavior of Human Epidermal Membranes. Journal of the Society of Cosmetic Chemists, 34, 21-34.

[29] Ananthapadmanabhan, K.P., Moore, D.J., Subramanyan, K., et al. (2004) Cleansing without Compromise: The Impact of Cleansers on the Skin Barrier and the Technology of Mild Cleansing. Dermatologic Therapy, 17, 16-25. https://doi.org/10.1111/j.1396-0296.2004.04S1002.x

[30] Putterman, G.J., Wolejsza, N.F., Wolfram, M.A. and Laden, K. (1997) The Effect of Detergents on Swelling of Stratum Corneum. Journal of the Society of Cosmetic Chemists, 28, 521-532.

[31] Moore, P.N., Puvvada, S. and Blankschtein, D. (2003) Challenging the Surfactant Monomer Skin Penetration Model: Penetration of Sodium Dodecyl Sulfate Micelles into the Epidermis. Journal of Cosmetic Science, 54, 29-46. 
[32] Damien, F. and Boncheva, M. (2010) The Extent of Orthorhombic Lipid Phases in the Stratum Corneum Determines the Barrier Efficiency of Human Skin in Vivo. Journal of Investigative Dermatology, 130, 611-614. https://doi.org/10.1038/jid.2009.272

[33] Li, J., Dahanayake, M., Reierson, R.L. and Tracy, D.J. (1999) Amphoteric Surfactants Having Multiple Hydrophobic and Hydrophilic Groups. U.S. Patent 5914310.

[34] Myers, D. (2006) Surfactant Science and Technology. 3rd Edition, John Wiley \& Sons, Inc., Hoboken, NJ. https://doi.org/10.1002/047174607X

[35] Agner, T. and Serup, J. (1990) Sodium Lauryl Sulphate for Irritant Patch Testing-A Dose-Response Study Using Bioengineering Methods for Determination of Skin Irritation. Journal of Investigative Dermatology, 95, 543-547.

[36] Ghosh, S. and Blankschtein, D. (2007) Why Is Sodium Cocoyl Isethionate (SCI) Mild to the Skin Barrier?-An in Vitro Investigation Based on the Relative Sizes of the SCI Micelles and the Skin Aqueous Pores. Journal of Cosmetic Science, 58, 229-244.

[37] Draelos, Z., Hornby, S., Walters, R.M. and Appa, Y. (2013) Hydrophobically Modified Polymers Can Minimize Skin Irritation Potential Caused by Surfactant-Based Cleansers. Journal of Cosmetic Dermatology, 12, 314-321.

https://doi.org/10.1111/jocd.12061

[38] Ananthapadmanabhan, K.P. (1993) Protein-Surfactant Interactions. In: Goddard, E.D., Ed., Interactions of Surfactants with Polymers and Proteins, CRC Press, Boca Raton, 319. https://doi.org/10.1201/9781351073783-9

[39] Janssens, M., van Smeden, J., Gooris, G.S., et al. (2011) Lamellar Lipid Organization and Ceramide Composition in the Stratum Corneum of Patients with Atopic Eczema. Journal of Investigative Dermatology, 131, 2136-2138. https://doi.org/10.1038/jid.2011.175

[40] Lemery, E., et al. (2015) Surfactants Have Multi-Fold Effects on Skin Barrier Function. European Journal of Dermatology, 25, 424-435. https://doi.org/10.1684/ejd.2015.2587

[41] Fluhr, W., Bornkessel, A. and Berardesca, E. (2006) Glycerol Just a Moisturizer? Biological and Biophysical Effects. In: Loden, M. and Maiback, H.I., Eds., Dry Skin and Moisturizers, CRC Press, Boca Raton, FL, 227-244.

[42] Moore, P.N., Puvvada, S. and Blankschtein, D. (2003) Role of the Surfactant Polar Head Structure in Protein-Surfactant Complexation: Zein Protein Solubilization by SDS and by SDS/C ${ }_{12} \mathrm{E}_{n}$ Surfactant Solutions. Langmuir, 19, 1009-1016. https://doi.org/10.1021/la020613k 